\title{
Vinculación entre el Ordenamiento Territorial y la gestión del agua en Argentina y en la provincia de Buenos Aires. Análisis de aspectos normativos e institucionales (2003-2019)
}

\section{Link between Spatial Planning and water management in Argentina and in the province of Buenos Aires. Analysis of regulatory and institutional aspects (2003-2019)}

\author{
Gabriela Calderon ${ }^{1}$ (), María Laura Zulaica² ${ }^{\circledR}$, \\ Héctor Enrique Massone ${ }^{3}$ (ib y Julieta Dalla Torre ${ }^{4}$ (i)
}

\begin{abstract}
RESUMEN
El Ordenamiento Territorial (OT) y la Gestión Integrada de Recursos Hídricos (GIRH), conforman instrumentos centrales para alcanzar los Objetivos de Desarrollo Sostenible (ODS) previstos en la Agenda 2030. Aunque existe un reconocimiento de la importancia de articular estas políticas, su vinculación ha sido escasamente estudiada. El presente trabajo analiza la vinculación de los aspectos normativos e institucionales entre las políticas públicas de OT y de gestión del agua en Argentina y en la Provincia de Buenos Aires (PBA) en el período 2003-2019. El procedimiento metodológico incluyó la revisión de aspectos relevantes de dichas políticas a nivel nacional y provincial, con énfasis en las principales normas y planes propuestos. A nivel nacional se destaca la ausencia de leyes en ambas temáticas; así como los escasos puntos en común entre estas políticas, con la única excepción de la gestión del riesgo. En la PBA existen dos normas que condicionan fuertemente el OT y la gestión del agua a nivel local, siendo su relación débil. La investigación realizada permite destacar la importancia de vincular estos procesos desde una perspectiva integral a fin de contribuir a la sostenibilidad en el marco de las metas previstas en los ODS.
\end{abstract}

Palabras clave: política pública; planificación territorial; Gestión Integrada de Recursos Hídricos; Argentina

Consejo Nacional de Investigaciones Científicas y Técnicas (CONICET). Instituto del Hábitat y el Ambiente (IHAM), Facultad de Arquitectura, Urbanismo y Diseño (FAUD), Universidad Nacional de Mar del Plata (UNMdP). Mar del Plata, Argentina.

Correo electrónico: gabicalderon.arq@gmail.com

CONICET. IHAM, FAUD, UNMdP. Grupo de Estudios Sociourbanos, Facultad de Humanidades (FH), UNMdP. Mar del Plata, Argentina. Correo electrónico: laurazulaica@conicet.gov.ar

Instituto de Geología de Costas y del Cuaternario (IGCyC), Facultad de Ciencias Exactas y Naturales (FCEyN), UNMdP / Comisión de Investigaciones Científicas de la Provincia de Buenos Aires (CIC). Mar del Plata, Argentina. Correo electrónico: massoneh@gmail.com CONICET. Instituto Multidisciplinario de Estudios Sociales Contemporáneos (IMESC) / Instituto de Estudios Históricos, Económicos, Sociales e Internacionales (IDEHESI). Facultad de Ciencias Políticas y Sociales (FCPyS), Universidad Nacional de Cuyo (UNCuyo). Mendoza, Argentina. Correo electrónico: julietadallatorre@gmail.com 


\begin{abstract}
Spatial Planning (SP) and Integrated Water Resources Management (IWRM) constitute central instruments to achieve the Sustainable Development Goals (SDGs) foreseen in the 2030 Agenda. Although there is a recognition of the importance of linking these policies, their connection has been scarcely studied. This work analyzes the link of normative and institutional aspects between SP and water management public policies in Argentina and the Buenos Aires Province (BAP) in the period 2003-2019. The methodological procedure included the review of relevant aspects of these policies at the national and provincial level, with emphasis on the main laws and proposed plans. At the national level, the absence of laws on both issues stands out; as well as the few points in common between them, with the exception of risk management. In the BAP there are two norms that strongly condition SP and water management at the local level, being their relationship weak. The research carried out makes it possible to highlight the importance of linking these processes from a comprehensive perspective in order to contribute to sustainability within the framework of the SDGs.
\end{abstract}

Keywords: public policy; spatial planning; Integrated Water Resources Management; Argentina

La relación entre la ocupación del territorio y el agua se hace evidente a partir de procesos que garantizan la vida de las personas y el desarrollo de sus actividades (agrícolas, ganaderas, forestales, mineras, industriales, recreativas, procesos de urbanización, entre otras). Sin embargo, dichas actividades pueden afectar de una u otra forma a los recursos hídricos, originando procesos tales como: la contaminación de origen residencial, agrícola o industrial; la sobreexplotación de las fuentes de recursos hídricos; las inundaciones/anegamientos; la eutrofización de los cuerpos de agua; la erosión, etc. (Carter, 2007). De ahí la necesidad de una adecuada vinculación entre la gestión hídrica y la gestión territorial, recurriendo a prácticas sostenibles en todos los usos del suelo y las actividades que se desarrollen en las cuencas hidrográficas, a fin de prevenir riesgos de origen hídrico, así como garantizar el acceso al agua para el consumo humano, una de las claves para lograr el Desarrollo Sostenible (Dourojeanni \& Jouravlev, 2001; Pochat, 2008; WWAP, 2019).

En efecto, el sexto Objetivo de Desarrollo Sostenible (ODS 6), definido en la Agenda 2030 por la Organización de las Naciones Unidas (ONU) en 2015, prevé "garantizar la disponibilidad de agua y su gestión sostenible y el saneamiento para todos". A su vez, el ODS 11, plantea "lograr que las ciudades y los asentamientos humanos sean inclusivos, seguros, resilientes y sostenibles", y ello demanda, entre otras cuestiones, tender a procesos de urbanización inclusivos, incrementando la capacidad para la planificación y la gestión participativa, integrada y sostenible de los asentamientos humanos. En este sentido, tanto el Ordenamiento Territorial (OT) como la Gestión Integrada de Recursos Hídricos (GIRH) conforman instrumentos centrales para alcanzar los ODS.

Autores europeos resaltan el aumento de la interacción entre la gestión del agua y el OT, especialmente a partir de la sanción de la Directiva Marco sobre el Agua, principal instrumento de política hídrica en Europa (Wiering \& Immink, 2006; Kidd, 2007). Esta directiva, aprobada en 2000 por el Parlamento Europeo, introdujo un enfoque global para la gestión y la protección de las aguas superficiales y subterráneas centrado en las cuencas hidrográficas. En este sentido, del Moral Ituarte (2009) destaca que durante los últimos años se ha ido reforzando la idea de que la gestión del agua debe plantearse necesariamente en el marco de una estrategia territorial de referencia; que la planificación hidrológica tiene que entenderse como un instrumento al servicio de una 
determinada política territorial; y que las demandas y disponibilidades de agua de cada cuenca sólo pueden fundamentarse en el diagnóstico y la consiguiente formulación de la política de OT.

Por su parte, autores latinoamericanos como Ovalles et al. (2008) también destacan que es indispensable la incorporación del territorio en el diseño de políticas públicas basadas en el enfoque del Desarrollo Sostenible y que la ordenación de cuencas hidrográficas es una herramienta apropiada al servicio de esta concepción de desarrollo. Asimismo, Andrade Pérez \& Navarrete Le Blas (2004) plantean que es cada vez más urgente integrar la gestión del agua a la planificación de los usos del suelo y el OT, permitiendo evaluar de manera integral y equitativa, la distribución y el manejo del agua en todo el ciclo hidrológico, trascendiendo las fronteras político-administrativas a nivel municipal, regional y hasta global.

Es indudable que el agua es determinante y limitante a la hora de plantear cualquier proceso de gestión territorial, por lo que no es posible la concepción ni la aplicación de una legislación de OT que esté disociada de la gestión integrada del agua (Duek \& Comellas, 2011). Asimismo, tampoco es factible plantear un proceso de GIRH en un territorio determinado, sin una clara referencia a las relaciones e interacciones que se producen entre los distintos componentes del sistema territorial en cuestión (Vich, 2010). En esta línea, Gudiño \& Cuello Rüttler (2017) destacan la importancia de considerar al OT como una forma de planificación y gestión que contemple a los recursos hídricos como un componente vital de la organización del territorio. No obstante la importancia de estos procesos existen miradas críticas en relación a su implementación, particularmente en cuanto a la GIRH. Al respecto, pueden consultarse, entre otros aportes: Biswas (2008), Molle (2006; 2012), Molle \& Wester (2009) y Delgadillo \& Durán (2012).

Aunque existe un reconocimiento de la importancia de articular las políticas hídricas y las territoriales, éstas han estado tradicionalmente separadas o con escasos puntos de encuentro (Woltjer \& Al, 2007). Esta tendencia se profundiza en el contexto latinoamericano, donde se observa un desigual abordaje en el ordenamiento de las cuencas hidrográficas (Dourojeanni et al., 2002). Como antecedente normativo en Argentina cabe destacar la consideración de la GIRH en la Ley 8.051/2009 de OT y Usos del Suelo de Mendoza. En este sentido, el Plan Provincial de OT (2017) se propone, entre otros objetivos, impulsar la GIRH como elemento estratégico para el OT, propendiendo a la preservación del recurso, la eficiencia de su uso y la garantía de su acceso para el consumo humano y las actividades productivas.

Sin embargo, la vinculación entre estos procesos ha sido escasamente analizada y profundizada en el marco político-institucional argentino. Por lo tanto, el presente trabajo ${ }^{5}$ se propone analizar la vinculación de los aspectos normativos e institucionales entre las políticas públicas de OT y de gestión del agua en Argentina y en la Provincia de Buenos Aires (PBA) en el período 2003-2019.

El artículo fue elaborado sobre la base de resultados correspondientes a la tesis de Doctorado en Ordenamiento Territorial y Desarrollo Sostenible (Universidad Nacional de Cuyo) de la primera autora del trabajo. 


\section{Territorios en análisis y principales aspectos teórico- metodológicos}

Argentina es un país federal constituido por 23 provincias y la Ciudad Autónoma de Buenos Aires (CABA), que se caracteriza por una amplia diversidad ecológica reflejada en una matriz productiva diversa, y por marcados desequilibrios territoriales. De acuerdo con proyecciones realizadas por el Instituto Nacional de Estadísticas y Censos, la población en 2020 alcanzaría 45.376.763, siendo más del $90 \%$ urbana y cuya distribución en el territorio es desigual.

La PBA se encuentra en la región pampeana y abarca el 8,1\% del territorio nacional. Está dividida en 135 partidos (o municipios) y es la provincia más poblada reuniendo aproximadamente el $40 \%$ de la población total del país, concentrada principalmente en el Área Metropolitana de Buenos Aires (AMBA, incluye CABA y 40 municipios provinciales).

La gestión de recursos hídricos es un tema recurrente en las políticas públicas provinciales y motivo de numerosos planes hidráulicos (Dirección Provincial de Saneamiento y Obras Hidráulicas, 2009). En el AMBA los planes históricos condensan los modos de pensar y de operar sobre el territorio en áreas con fuertes procesos de urbanización expuestas a inundaciones (Potocko, 2017). Por otra parte, el Plan Maestro Integral del Río Salado, iniciado en 1997, da cuenta de la problemática regional de una cuenca que abarca casi las dos terceras partes de la superficie total de la Provincia. Sus condiciones topográficas acompañadas por cíclicas variaciones climáticas inducen periódicas y recurrentes inundaciones y sequías prolongadas que afectan la producción y la economía de la región. Asimismo, esta llanura ha registrado en los últimos 50 años un fuerte ascenso en los niveles freáticos asociado al incremento de precipitaciones y a los cambios en el uso del suelo (García et al., 2018). La complejidad territorial, jurisdiccional y las problemáticas de la región derivan en conflictos de difícil resolución. A su vez, la dinámica hídrica incide no sólo en el desarrollo de las actividades productivas sino también en los modos de habitar las ciudades (Ruiz et al., 2019).

En este contexto, la PBA fue la primera en contar con una normativa específica de OT, el Decreto-Ley de OT y Uso del Suelo (DL 8.912/1977), junto con el Código de Planeamiento Urbano de la ciudad de Buenos Aires del mismo año ${ }^{6}$. Las particularidades señaladas justifican el interés de abordar este territorio.

Por otra parte, a nivel nacional, con la última reforma constitucional (1994) se incorporaron los derechos de incidencia colectiva en el ordenamiento jurídico argentino y se modificó parcialmente la distribución de competencias entre la Nación y las provincias en aquellos aspectos atinentes a la gestión de los recursos naturales. Además, la Constitución Nacional (CN), incluye en el Artículo 41 el derecho a un ambiente sano, equilibrado y apto para el desarrollo humano, principios básicos tanto para el OT como para la GIRH.

Al 2019 sólo cuatro jurisdicciones contaban con leyes en materia de OT: PBA, Mendoza (Ley 8.051/2009 de OT y Usos del Suelo), Jujuy (Ley 6.099/2018 de Ordenamiento Territorial, Uso y Fraccionamiento del Suelo) y CABA (Código Urbanístico aprobado en 2018). 
En el mismo año, la Constitución de la PBA incorporó estos mismos derechos en el Artículo 28. En virtud de esta incorporación se sancionó la Ley Integral del Medio Ambiente y los Recursos Naturales de la PBA 11.723/1996 que prevé el OT entre los instrumentos de gestión ambiental y, posteriormente, el Código de Aguas (Ley 12.257/1999). No obstante la existencia de estos instrumentos, la gestión de los recursos hídricos ha sido históricamente afrontada desde una perspectiva sectorial.

El recorte temporal de esta investigación abarca principalmente el período comprendido entre los años 2003 y 2019, que incluye las presidencias de Néstor Kirchner (2003-2007), Cristina Fernández de Kirchner (2007-2015) y Mauricio Macri (2015-2019).

EI OT se considera un proceso sujeto a los procedimientos que incluyen actividades de diagnóstico y prospectiva territorial, formulación de objetivos, elaboración de los lineamientos estratégicos y determinación de las acciones por realizar, así como la implementación de dichas acciones (Massiris Cabeza, 2012). Según el autor, el OT se concreta en planes que expresan el modelo territorial a largo plazo que la sociedad percibe como deseable y las estrategias mediante las cuales se actuará sobre la realidad para evolucionar hacia dicho modelo.

De acuerdo con la Asociación Mundial por el Agua (Global Water Partnership, GWP), la GIRH es un proceso que promueve el desarrollo y la gestión coordinados del agua, la tierra y los recursos relacionados, con el fin de maximizar el bienestar social y económico resultante de manera equitativa sin comprometer la sostenibilidad de los ecosistemas vitales (GWP, 2000; GWP, 2009). Este enfoque busca contribuir en la administración de los recursos hídricos de manera equitativa, participativa, eficiente y sostenible, teniendo en cuenta los intereses sociales, económicos y ecosistémicos. En este sentido, reconoce los diferentes actores que compiten entre sí, los sectores que usan y abusan del agua y las necesidades de los ecosistemas y busca coordinarlos para lograr acciones efectivas; para ello, la GIRH propone alrededor de 30 "instrumentos de gestión", alguno de los cuales son utilizados desde hace tiempo, como la evaluación de impacto ambiental, la evaluación de servicios ecosistémicos y la evaluación y gestión del riesgo hídrico (GWP, 2009).

La trayectoria de las políticas públicas en materia de OT y gestión del agua en Argentina y en la PBA, ha atravesado diferentes momentos. En ese sentido, una política pública es, esencialmente, una toma de posición del Estado frente a una determinada cuestión socialmente problematizada (Thwaites Rey, 2005). De esta manera, se define a la política pública como una toma de posición, tácita o explícita, de diferentes agencias e instancias del aparato estatal que expresan una determinada modalidad de intervención del Estado, en relación con una cuestión que despierta la atención, interés o movilización de actores de la sociedad civil (Oszlak \& O’Donnell, 1981).

Partiendo de este enfoque, el procedimiento metodológico utilizado incluyó la revisión de aspectos relevantes de las políticas de OT y de gestión del agua a nivel nacional y provincial, con énfasis en las principales normas y planes propuestos. Además, se estudiaron las leyes más significativas a nivel provincial, a fin de identificar vínculos o puntos en común. Por otra parte, se enunciaron los principales agentes públicos intervinientes en ambas esferas.

Las fuentes documentales consultadas fueron las siguientes: las normas nacionales y provinciales (Constitución Nacional y Provincial, tratados internacionales, leyes, decretos, anteproyec- 
tos de ley); los planes de gestión (principios rectores, planes estratégicos y planes de obras); los documentos oficiales publicados (objetivos, rol o misión de los organismos, organigramas); y los artículos periodísticos que anunciaran reuniones o encuentros entre actores, así como también cambios en las estructuras organizativas de los organismos del Estado. Además, se consultaron las páginas web de los organismos públicos que estuvieran disponibles.

\section{Aspectos normativos e institucionales de las políticas públicas en materia de OT en Argentina y la PBA}

De acuerdo con Massiris Cabeza (2012), las políticas de OT se iniciaron en América Latina a finales de los años sesenta ligadas a la regulación del uso del suelo urbano. Sin embargo, el resultado general de estos procesos ha sido modesto en cuanto al objetivo de regular y controlar el uso y la ocupación de los territorios, generando desequilibrios territoriales, inequidades sociales y deterioro ambiental. Entre sus causas, el autor menciona el predominio de las visiones sectoriales y los intereses de los actores influyentes, la ausencia de voluntad política para el cumplimiento de los planes, la débil participación social en el proceso y la calidad de la información.

En Argentina, durante el período de gestión 2003-2007, se promulgó la Política Nacional de Desarrollo y OT Argentina 2016, la cual se enfocó en guiar y orientar las acciones con impacto territorial hacia el desarrollo equilibrado, integrado, sustentable y socialmente justo del territorio argentino, que supere las concepciones sectoriales y permita aprovechar las oportunidades de desarrollo. En articulación con esta política, el Ministerio de Planificación Federal, Inversión Pública y Servicios (MINPLAN), a través de la Subsecretaría de Planificación Territorial de la Inversión Pública, formuló el Plan Estratégico Territorial (PET) en el año 2004. Mediante este instrumento, se intentó orientar el uso y ocupación del territorio con el objetivo de: 1) mejorar y sostener el crecimiento de la producción en forma equilibrada mediante la construcción de infraestructura y equipamiento; 2) garantizar el acceso de la población a los bienes y servicios básicos, promoviendo el desarrollo equitativo de las regiones y el arraigo de sus habitantes; 3) contribuir a la valorización del patrimonio natural y cultural a través de una gestión integrada y responsable (Massiris Cabeza, 2012).

Dando continuidad a esta política, en el período de gestión 2007-2015, se conformó el Consejo Federal de Planificación y OT (COFEPLAN), el cual articula la acción de la Nación, los gobiernos provinciales y CABA. En 2012, el COFEPLAN elaboró el Anteproyecto de Ley Nacional de Planificación y OT, el cual se sumó a otros proyectos de ley vinculados a esta temática presentados en el Congreso Nacional en los años 2009, 2011, 2013 y 2018. A pesar de esto, todavía no se dictó una ley de OT a nivel nacional, por lo que este debate continúa abierto7. Además, se presentaron avances del PET en los años 2008, 2010, 2012 y 2015.

A partir de diciembre de 2015, el MINPLAN fue disuelto y su jerarquía descendió, incluyéndose en la estructura del Ministerio del Interior, Obras Públicas y Vivienda (MIOPyV), bajo el nombre de Secretaría de Planificación Territorial y Coordinación de Obra Pública. Durante el período 2015-

Se destaca la existencia de leyes nacionales de presupuestos mínimos que contienen políticas implícitas de OT como es el caso de la Ley General del Ambiente (25.675/2002) o la Ley de Bosques Nativos (26.331/2007). 
2019, el COFEPLAN continuó reuniéndose en asambleas y se desarrolló un nuevo avance en el PET (2018) incorporando los compromisos asumidos a nivel internacional, como los ODS, los acuerdos del Marco de Sendai en relación con la gestión de riesgos de desastres y la Nueva Agenda Urbana acordada en la Conferencia de las Naciones Unidas sobre la Vivienda y el Desarrollo Urbano Sostenible, "Hábitat III" (Quito, 2016). Además, el MIOPyV, en conjunto con ONU-Hábitat, formuló la Política Nacional Urbana Argentina (2018), basada en nueve ejes, entre ellos: gestión integral, planificación urbana y OT; equipamiento e infraestructura urbana integrados; gestión ambiental, resiliencia y cambio climático; y gobernanza local efectiva. También se presentaron las guías para la elaboración de normativa de OT a escala provincial y para la elaboración de normativa urbana (2019), donde se destaca la importancia de considerar las áreas de riesgo.

En el ámbito de la PBA, además de la Constitución Provincial, existen diferentes normas generales que encuadran la política de OT: la Ley Orgánica de las Municipalidades (LOM, DL 6.769/1958 y modificaciones), el Código Rural (DL 10.081/1983 y modificaciones) y la Ley de Catastro Territorial (Ley $10.707 / 1988$ y modificaciones).

La sanción del DL 8.912/1977 de OT y Usos del Suelo, decretado durante la última dictadura cívico-militar y aún en vigencia, estuvo urgida por la necesidad de contener el irracional proceso de subdivisión de tierras sin infraestructuras y en situaciones de riesgo que venía efectuándose en la provincia (Ríos et al., 2008). La ley, compuesta por seis Títulos, regula el uso, ocupación, subdivisión y equipamiento del suelo (Artículo 1, DL 8.912/1977) y define los objetivos del OT en su Artículo 2.

En cuanto a sus principios, se establece que el OT debe ser concebido "como un proceso ininterrumpido en el que un conjunto de pautas y disposiciones normativas orienten las decisiones y acciones del sector público y encaucen las del sector privado, hacia el logro de objetivos predeterminados". Además, los municipios deben "realizarlo en concordancia con los objetivos y estrategias definidas por el Gobierno Provincial para el sector y con las orientaciones generales y particulares de los planes provinciales y regionales de desarrollo económico y social y de ordenamiento físico" (Artículo 3, DL 8.912/1977).

Asimismo, la provincia atribuye a los municipios la responsabilidad de la organización de sus respectivos territorios. Por consecuencia, a través de sus Concejos Deliberantes, los municipios disponen de facultades expresamente delegadas por la Provincia para la implementación del OT y del control del uso del suelo. Esto permite que los municipios asuman un rol protagónico en materia de OT. Además, por una cuestión de proximidad, los gobiernos locales se encuentran en condiciones más propicias que otros niveles gubernamentales para reglamentar el ordenamiento de sus territorios.

Por otra parte, esta norma determina cuatro etapas del proceso de planeamiento a nivel municipal: delimitación preliminar de áreas, zonificación, plan de ordenamiento urbano y planes particularizados. Sin embargo, son escasos los municipios que alcanzaron a cumplir con todas las etapas mencionadas y muchos aún se encuentran en la delimitación preliminar de áreas (Corti, 2009).

La implementación del DL 8.912 trajo consigo el encarecimiento de la tierra y, como corolario, el crecimiento de la informalidad urbana al no prever mecanismos que garanticen el acceso al suelo urbanizado de aquellos sectores que no pueden afrontar sus costos (Clichevsky, 2003). 
En contrapartida, se sancionó en 2012 la Ley de Acceso Justo al Hábitat (14.449/2012) que incorpora la complejidad del déficit urbano habitacional sostenida en cuatro principios: el derecho a la ciudad y a la vivienda, la función social de la propiedad, la gestión democrática de la ciudad, y el reparto equitativo de las cargas y los beneficios. Sus objetivos específicos son: a. Promover la generación y facilitar la gestión de proyectos habitacionales, de urbanizaciones sociales y de procesos de regularización de barrios informales; b. Abordar y atender integralmente la diversidad y complejidad de la demanda urbano habitacional: c. Generar nuevos recursos a través de instrumentos que permitan reducir las expectativas especulativas de valorización del suelo. Esta ley modifica algunos Artículos del DL 8.912, al tiempo que la complementa con respecto a la gestión integral del hábitat y el derecho a un hábitat digno. En términos generales, hasta el momento, la Ley se encuentra en un proceso reciente de implementación y ha tenido avances diferenciales en el ámbito municipal.

Con respecto a los instrumentos de gestión territorial, en 2006, el Ministerio de Infraestructuras, Viviendas y Servicios Públicos (MIVySP) realizó un diagnóstico denominado "Dinámica espacial actual del territorio bonaerense" a raíz del proceso iniciado con el PET nacional. Además, en 2007 se desarrollaron los Lineamientos Estratégicos para la Región Metropolitana de Buenos Aires entre la Subsecretaría de Urbanismo y Vivienda de la PBA y la Secretaría de Planeamiento de la CABA. Más adelante, el Ministerio de Infraestructura publicó el Plan Estratégico Territorial de la PBA, el cual constituye una lista de proyectos de infraestructura para la provincia. Sin embargo, aún no se ha formulado un plan de OT a nivel provincial.

\section{Aspectos normativos e institucionales de las políticas públicas en materia de gestión del agua en Argentina y la PBA}

En cuanto a la gestión de los recursos hídricos, en casi todos los países de América Latina y el Caribe se pusieron en marcha reformas legislativas vinculadas a la gestión y el aprovechamiento del agua (Jouravlev, 2001), especialmente a partir de la década de los ‘90. Esto estuvo enmarcado por la emergencia y expansión a nivel internacional de la GIRH y fue motivado tanto por una percepción de degradación creciente de los recursos como por una insatisfacción con los modelos y prácticas de gestión preexistentes (Gutiérrez, 2011). A pesar de las diferencias entre los países de la región, Jouravlev (2001) destaca puntos en común como la reducción del rol del Estado, la descentralización de responsabilidades hacia los gobiernos locales, el interés por implementar instrumentos económicos en la gestión de los recursos hídricos y la incorporación del sector privado y los usuarios en la gestión.

En cuanto a la política hídrica en Argentina, la CN establece en el Artículo 124 que le "corresponde a las provincias el dominio originario de los recursos naturales existentes en su territorio". Varios autores coinciden en que esto ha llevado a la ausencia de una legislación hídrica propiamente nacional y a una alta heterogeneidad de legislaciones provinciales y de organizaciones sectoriales (Jouravlev, 2001; Gutiérrez, 2011). En este sentido, el predominio de enfoques sectoriales que se multiplican, diversifican y fragmentan a través de las provincias, acarrea fallas de coordinación, superposición de funciones, dilución de responsabilidades y ocurrencia de conflictos intersectoriales e interjurisdiccionales (Gutiérrez, 2011). 
A su vez, desde 1996 se comenzó a dar cumplimiento al requerimiento de los presupuestos mínimos contemplados en el Artículo 41 de la CN que establece, entre otras cuestiones, la importancia de que las actividades productivas satisfagan las necesidades presentes sin comprometer las de las generaciones futuras, señalando que corresponde a la Nación dictar las normas que contengan los presupuestos mínimos de protección ambiental, y a las provincias, las necesarias para complementarlas.

En este sentido, la Ley General del Ambiente (25.675/2002), destaca en su Artículo 6 la importancia de prever las condiciones necesarias para garantizar la dinámica de los sistemas ecológicos, mantener su capacidad de carga y asegurar la preservación ambiental y el desarrollo sustentable, proponiendo en su Artículo 8, al "ordenamiento ambiental del territorio" como uno de sus instrumentos de política y gestión ambiental, entendido éste como un proceso que tiene en cuenta los aspectos políticos, físicos, sociales, tecnológicos, culturales, económicos, jurídicos y ecológicos de la realidad local, regional y nacional. Posteriormente, se sancionó la ley 25.688/2002, denominada Régimen de Gestión Ambiental de Aguas, la cual nunca fue reglamentada. Esta ley fue sujeto de numerosas críticas y presentaciones judiciales que impulsaban su inconstitucionalidad, y que entendían que la ley avanzaba sobre competencias provinciales no delegadas a la Nación en materia de cuencas y de organización de Comités de Cuencas, como también en la gestión de los recursos naturales, el desarrollo de instituciones locales y la planificación, uso y gestión del agua (Pochat, 2008). Diversos proyectos de ley se encuentran todavía en proceso de discusión y debate.

Por otro lado, a partir del año 2000, la Subsecretaría de Recursos Hídricos de la Nación (SSRH) promovió un proceso de discusión con sus pares provinciales que culminó en 2003 con la definición de los Principios Rectores de la Política Hídrica (PRPH) en el seno del Consejo Hídrico Federal (COHIFE). Este consejo, constituido en 2003, es una instancia federal integrada por los Estados provinciales (incluida la CABA) y por el Estado nacional a través de la SSRH para la concertación y coordinación de la política hídrica federal. Los 49 principios organizados en torno a 8 dimensiones tienen por objetivo generar lineamientos tendientes a la integración de los aspectos técnicos, sociales, económicos, legales, institucionales y ambientales del agua a nivel nacional. Por su parte, el Principio Rector 5, "Articulación de la gestión hídrica con la gestión territorial", permite dar cuenta de la importancia de la incorporación de la gestión del agua al OT y el Principio Rector 8 deja explicitado el derecho humano al agua potable y al saneamiento. En 2005, los PRPH fueron presentados como anteproyecto de ley ante el Congreso de la Nación, pero éste nunca fue tratado (Gutiérrez, 2011).

Posteriormente, en 2007, la SSRH formuló un documento base del Plan Nacional Federal de Recursos Hídricos orientado a compatibilizar las acciones contempladas en las planificaciones provinciales, regionales, sectoriales y por cuencas, pero sin proponerse objetivos, metas cuantificables o estrategias de implementación. Esto fue destacado en una evaluación llevada a cabo en 2015 por la Auditoría General de la Nación.

Los cambios políticos que se generaron a partir de diciembre de 2015 también significaron cambios en la política hídrica. A partir de 2016, la SSRH, que ascendió su jerarquía y cambió de nombre a Secretaría de Infraestructura y Política Hídrica del MIOPyV, puso en funcionamiento el Plan Nacional del Agua, un plan de obras que se propone la cobertura universal de agua potable, 
la provisión de saneamiento, la reducción de la vulnerabilidad frente a los extremos climáticos, el incremento de la superficie bajo riego y el desarrollo de proyectos de propósitos múltiples. Dicho plan se apoya en ejes centrales de la política hídrica y, contempla otros ejes transversales como son la preservación del recurso hídrico, el fortalecimiento de capacidades, la innovación y la participación. El único instrumento vinculado a los PRPH que menciona este documento es el Principio 20, que hace referencia a los planes estratégicos provinciales. Por su parte, el Comité Ejecutivo del COHIFE continuó reuniéndose periódicamente.

Por otra parte, en 2016, se sanciona la Ley 27.287/2016 que crea el Sistema Nacional para la Gestión Integral del Riesgo (SINAGIR), el cual constituye uno de los instrumentos de la GIRH, mencionados anteriormente. La ley tiene por objetivo integrar acciones y articular el funcionamiento de los organismos del gobierno nacional, los gobiernos provinciales, de la CABA y municipales, las organizaciones no gubernamentales y la sociedad civil, para fortalecer y optimizar las acciones destinadas a la reducción de riesgos, el manejo de la crisis y la recuperación. El SINAGIR tiene como finalidad la protección integral de las personas, las comunidades y el ambiente ante la existencia de riesgos.

En cuanto a los recursos naturales de la PBA, la Constitución Provincial señala, en el ya mencionado Artículo 28, que "la Provincia ejerce el dominio eminente sobre el ambiente y los recursos naturales de su territorio incluyendo el subsuelo y el espacio aéreo correspondiente, el mar territorial y su lecho, la plataforma continental y los recursos naturales de la zona económica exclusiva, con el fin de asegurar una gestión, ambientalmente adecuada". Establece, entre otros aspectos, que "deberá preservar, recuperar y conservar los recursos naturales, renovables y no renovables, planificar el aprovechamiento racional de los mismos; controlar el impacto ambiental de todas las actividades que perjudiquen al ecosistema y promover acciones que eviten la contaminación del aire, agua y suelo".

En virtud de ello, se aprobó en 1995 la Ley de Protección del Medio Ambiente y Recursos Naturales (11.723/1995) que, al igual que la Ley General del Ambiente, propone al "ordenamiento ambiental" como uno de sus instrumentos de política ambiental. Además, de modo general, fija los principios que rigen "la implementación de políticas para la protección y mejoramiento del recurso agua", los cuales están vinculados a la GIRH. Asimismo, se establece que el tratamiento integral del recurso deberá efectuarse teniendo en cuenta las regiones hidrográficas y/o cuencas existentes en la PBA, a partir de la creación de comités de cuenca (Artículo 43, Ley 11.723/1995).

Posteriormente, en 1999, el Poder Legislativo provincial aprobó el Código de Aguas (Ley 12.257/1999), que contiene 80 Artículos, organizados en 12 Títulos. El mismo crea la Autoridad del Agua (ADA), autoridad de aplicación de la política hídrica provincial, un ente autárquico de naturaleza multidisciplinaria que tiene a su cargo la planificación, el registro, la constitución y la protección de los derechos, la policía y el cumplimiento y ejecución de lo establecido en el Código, cuya organización y funcionamiento deben estar basadas en la descentralización operativa y financiera (Artículo 3, Ley 12.257/1999).

El Código de Aguas establece los siguientes instrumentos: la planificación hidrológica para satisfacer las demandas de agua; el inventario físico; las cartas de riesgo hídrico de las zonas que pueden ser afectadas por inundaciones; los permisos y las concesiones para la ocupación, el uso o el aprovechamiento del agua; el canon por el uso del agua; las contribuciones para obras y ser- 
vicios; las evaluaciones de impacto ambiental; los comités de cuencas hídricas y los consorcios de usuarios. A excepción de los consorcios, la ADA es la autoridad de aplicación exclusiva de todos estos instrumentos, lo cual da cuenta de una fuerte centralización en la gestión del agua.

Asimismo, el Artículo 4 de la Ley destaca entre sus funciones la de coordinar un espacio interinstitucional con órganos provinciales competentes en materia de agua. Según el Decreto Reglamentario 3.511/2007, esta Comisión de Coordinación Interinstitucional debe reunir a representantes de ADA, de la Secretaría de Política Ambiental (posteriormente Organismo Provincial para el Desarrollo Sostenible, OPDS), del Ministerio de Asuntos Agrarios, de la Dirección Provincial de Ordenamiento Urbano y Territorial (DPOUyT) y de la Dirección Provincial de Minería. Sin embargo, la conformación de esta comisión es aún incipiente.

Por otra parte, si bien se definen extensamente los múltiples usos del agua (consumo humano, abastecimiento de agua potable, uso agropecuario, uso industrial, uso recreativo, deportivo y de esparcimiento, uso energético, uso de aguas con propiedades terapéuticas, uso minero, uso ambiental, uso suntuario, flotación y navegación), es de destacar que en ningún momento define explícitamente a la cuenca hidrográfica como unidad territorial de gestión (Gutiérrez, 2011). De acuerdo con la misma fuente, prácticamente no se ven expresados en el Código varios de los principios de política hídrica formulados en la Ley 11.723/1995, en especial aquellos que plantean la descentralización operativa, la coordinación entre organismos involucrados en el manejo del recurso y la participación de usuarios.

Como otro hecho relevante de la política hídrica de la Provincia puede mencionarse que en 2003 se aprobó el Marco Regulatorio para la Prestación de los Servicios de Agua Potable y Desagües Cloacales (Decreto 878/2003), que crea el Organismo de Control del Agua de la PBA (OCABA), órgano de contralor de los prestadores del servicio.

En cuanto a instrumentos de planificación hídrica, en 2007 se presentó el Plan Hidráulico Provincial, enfocado en tres regiones: Área Metropolitana (Cuencas Río Luján, Río Reconquista, Matanza Riachuelo y Río de La Plata); Área Rural (Cuenca del Río Salado); y Área Costera Fluvial (Delta Bonaerense) y Marítima. Posteriormente, se presentó el Plan Estratégico de Agua y Saneamiento 2011-2041, con una profunda caracterización de los recursos hídricos de la provincia y de las problemáticas en cuanto al abastecimiento de agua potable y de desagües cloacales, entre otros. En este sentido, se mencionan como problemáticas vinculadas a la contaminación del agua: la falta de suministro de servicios cloacales que alteran la calidad del agua subterránea, la falta de planificación de usos del suelo en el desarrollo urbanístico, la ocupación desordenada del territorio, la insuficiencia de control por parte de los organismos responsables. El plan también propone cuatro ejes estratégicos que proponen reducir el déficit de agua y cloaca, optimizar y mejorar la calidad de los servicios de agua y saneamiento, llevar a cabo obras de infraestructura y diseñar planes regionales y de fortalecimiento institucional.

Un caso emblemático de gestión hídrica interjurisdiccional lo constituye la creación de la Autoridad de Cuenca Matanza Riachuelo (ACUMAR), en 2006 mediante la Ley Nacional 26.168/2006. ACUMAR es un ente autónomo, autárquico e interjurisdiccional que integra competencias de tres niveles: nacional, provincial y de CABA. Surgió como respuesta al deterioro ambiental de la Cuenca que en 2008 fue intimado por la Corte Suprema de Justicia de la Nación para implementar un plan de saneamiento en respuesta a una causa judicial (Causa Mendoza), a raíz del reclamo de vecinos 
organizados. En este contexto, se implementó en 2009 un Plan Integral de Saneamiento Ambiental (PISA), que tuvo diferentes fases en su desarrollo y puntos de conflicto que aún continúan, ligados sobre todo a la relocalización de villas ribereñas de la ciudad de Buenos Aires (Carman, 2019).

Posteriormente, en 2013 mediante el Decreto 429, que reglamenta el Artículo 43 del Código de Aguas, se establece al canon del agua -que deben pagar los concesionarios o permisionarios de derechos de uso de agua pública-, como el principal instrumento económico para solventar los Planes de Gestión Integrada de los Recursos Hídricos para cada región o subregión hidrológica en el ámbito provincial. Sin embargo, el cumplimiento de este canon es irregular y los avances en los planes insuficientes, aunque la Ley $14.782 / 2015$ reconoce el acceso al agua potable y al saneamiento como un derecho humano esencial para la vida.

En el marco de la reestructuración administrativa llevada a cabo desde 2016, el OCABA fue incorporado a la estructura organizativa de ADA, centralizando aún más sus funciones para desempeñar acciones específicas en el ámbito de actuación, sin que ello represente avances significativos en el ciclo de implementación.

\section{Políticas vigentes en materia de OT y gestión del agua: consideraciones sobre su vinculación}

La Figura $\mathrm{N}^{\circ} 1$ muestra una síntesis comparada de las políticas analizadas en cuanto a la normativa, los planes, programas y proyectos propuestos y los principales organismos del Estado Nacional y Provincial involucrados.

En el ámbito nacional, si bien se observa la ausencia de leyes en materia de OT y gestión del agua, se percibe un mayor avance en la política hídrica a partir del consenso alcanzado en la formulación de los PRPH. Por otra parte, es importante destacar los escasos puntos en común entre ambas políticas. El análisis de documentos, planes y encuentros entre actores destaca la importancia de la articulación entre la gestión del agua y la gestión del territorio. Sin embargo, no se avanzó en el diseño de instrumentos o acciones concretas tendientes a lograr esta vinculación a nivel institucional o normativo, con la única excepción de la gestión del riesgo.

A diferencia del nivel nacional, en la PBA existen dos normas que condicionan fuertemente el OT y la gestión del agua a nivel local: el DL 8.912/1977 y el Código de Aguas. Sin embargo, la relación entre ambas normas es débil, siendo apenas el Certificado de Aptitud Hidráulica uno de los pocos instrumentos que las vinculan de manera directa, como así también la iniciativa en el Código de Aguas y su Decreto Reglamentario para coordinar un espacio interinstitucional con los órganos de administración provincial, entre ellos la DPOUyT. Además, y aunque de manera más sutil, en el análisis de la articulación se pueden mencionar los Artículos 15 y 17 del DL 8.912/1977 que señalan que, para la creación y ampliación de núcleos urbanos o centros de población, se deberá demostrar la existencia de fuentes de aprovisionamiento de agua potable en calidad y cantidad para satisfacer las necesidades de la población potencial a servir. Por otra parte, el Artículo 173 del Código de Aguas señala que sólo se autorizarán fraccionamientos de tierras urbanas y suburbanas para vivienda en unidades cuya disponibilidad de agua potable alcance para abastecer a sus posibles habitantes. 
En términos generales, la política hídrica provincial contiene un marco normativo e institucional más robusto que la de OT. El principal organismo de aplicación (ADA) opera de manera muy centralizada y con pocos espacios de participación, por lo que la gestión del agua se encuentra lejos de ser integrada. Además, en general, los organismos de manejo y control del agua tienen una presencia limitada en el territorio municipal. Por otro lado, la responsabilidad sobre la gestión territorial está delegada a los municipios, quienes deben llevar a cabo sus planes de OT sin la existencia de un plan provincial de ordenamiento, tal como se observa en la Figura $\mathrm{N}^{\circ} 1$.

Figura $\mathrm{N}^{\circ} 1$

Normativas, instrumentos de gestión y organismos públicos de OT y de agua a nivel nacional y provincial.

\begin{tabular}{|c|c|c|c|}
\hline & & Ordenamiento Territorial & Gestión del agua \\
\hline \multirow{4}{*}{ 突 } & 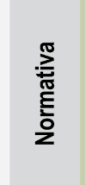 & $\begin{array}{l}\text { - Constitución Nacional: Art. } 41 \text { y Art. } 75 \text { inc. } 18 \text { y } 19 \\
\text { - Anteproyecto de Ley Nacional de Planificación y OT (2012) } \\
\text { - Varios proyectos presentados entre } 2009 \text { y } 2018\end{array}$ & $\begin{array}{l}\text { - Constitución Nacional: Art. 41. y Art. } 124 \\
\text { - Ley General del Ambiente (25.675/02) } \\
\text { - Régimen de Gestión Ambiental de Aguas (25.688/02) } \\
\text { (sin reglamentar) } \\
\text { - Observación General } 15 \text { del Comité de Derechos Económicos, } \\
\text { Sociales y Culturales (DESC) (2002) }\end{array}$ \\
\hline & 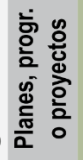 & $\begin{array}{l}\text { - Política Nacional de Desarrollo y OT Argentina } 2016 \text { (2004) } \\
\text { - Plan Estratégico Territorial y avances (2004; 2008; 2010; } \\
\text { 2011; 2015; 2018) } \\
\text { - Politica Nacional Urbana (2018) } \\
\text { - Bases para el Desarrollo Territorial (2019) }\end{array}$ & $\begin{array}{l}\text { - Principios Rectores de Política Hídrica (2003) } \\
\text { - Plan Nacional Federal de Recursos Hídricos (2007) } \\
\text { - Plan Nacional del Agua (2016) }\end{array}$ \\
\hline & & $\begin{array}{r}\text { - Ministerio de Planificación Federal, Inversión Pública y Ser } \\
\text { Públicas y Vivienda de la } \mathrm{Na}\end{array}$ & $\begin{array}{l}\text { vicios (MINPLAN 2003-2015) / Ministerio del Interior, Obras } \\
\text { ación (MIOPyV 2015-2019) }\end{array}$ \\
\hline & 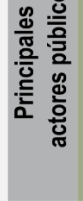 & $\begin{array}{l}\text { - Subsecretaría de Planificación Territorial de la Inversión } \\
\text { Pública (MINPLAN 2003-2015) / Secretaría de Planificación } \\
\text { Territorial y Coordinación de Obra Pública (MIOPyV 2015-2019) } \\
\text { - COFEPLAN }\end{array}$ & $\begin{array}{l}\text { - Subsecretaria de Recursos Hidricos (MINPLAN 2003-2015) / } \\
\text { Secretaria de Infraestructura y Politica Hídrica } \\
\text { (MIOPyV 2015-2019) } \\
\text { - Ente Nacional de Obras Hidricas de Saneamiento (ENOHSA) } \\
\text { - COHIFE } \\
\text { - Instituto Nacional del Agua (INA) } \\
\text { - } 14 \text { organismos de cuenca interprovinciales }\end{array}$ \\
\hline \multirow{4}{*}{ 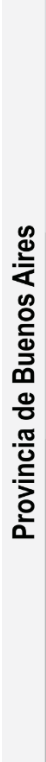 } & 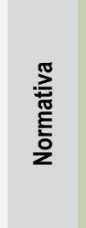 & $\begin{array}{l}\text { - Decreto-Ley de OT y Usos del Suelo ( } 8.912 / 77) \text {, } \\
\text { texto ordenando por Decreto ( } 3.389 / 87) \text { y modificatorias } \\
\text { - Ley de Acceso Justo al Hábitat (14.449/12) }\end{array}$ & $\begin{array}{l}\text { - Constitución Provincial: Art. } 28 \\
\text { - Ley de Protección del Medio Ambiente y Rec. Naturales (11.723/95) } \\
\text { - Código de Aguas (12.257/99), Dec. Reglam. (3.511/07) y modific. } \\
\text { - Marco Regulatorio para la Prestación de los Servicios Públicos de } \\
\text { Provisión de Agua Potable y Desagües Cloacales (11.820/96) } \\
\text { y Dec. Reglam. (878/03) } \\
\text { - Reconocimiento del Agua Potable y el Saneamiento como } \\
\text { Derecho Humano (14.782/15) }\end{array}$ \\
\hline & 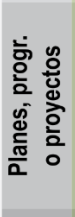 & $\begin{array}{l}\text { - Plan Estratégico Territorial de la PBA (2006) } \\
\text { - Lineamientos Estratégicos para la Región Metropolitana de Buenos } \\
\text { Aires (2007) } \\
\text { - Plan de Urbanización de Villas y Asentamientos Precarios en } \\
\text { Riesgo Ambiental de la Cuenca Matanza Riachuelo (2010) } \\
\text { - Plan particularizado de ordenamiento urbano y reconfiguración } \\
\text { para las márgenes de la Cuenca del Río Reconquista }\end{array}$ & $\begin{array}{l}\text { - Plan Hidráulico Provincial (2007) } \\
\text { - Plan Estratégico de Agua y Saneamiento (2011) } \\
\text { - Plan Maestro Integral Cuenca del Rio Salado (1997) } \\
\text { - Plan Integral de Saneamiento Ambiental Cuenca Matanza } \\
\text { Riachuelo (2009; 2016) }\end{array}$ \\
\hline & & $\begin{array}{r}\text { - Ministerio de Infraestructura, Vivienda y Servicios Públicos } \\
\text { Ministerio de Infraestructura y S }\end{array}$ & $\begin{array}{l}\text { s (2003-2007) / Ministerio de Infraestructura (2007-2015) I } \\
\text { Servicios Públicos (2015-2019) }\end{array}$ \\
\hline & 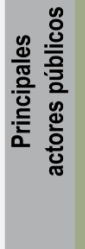 & $\begin{array}{l}\text { - Subsecretaria de Obras Públicas } \\
\text { - Dirección Provincial de Ordenamiento Urbano y Territorial } \\
\text { (DPOUyT) } \\
\text { - Gerencia General de Catastro y Geodesia de la Agencia de } \\
\text { Recaudación (ARBA) }\end{array}$ & $\begin{array}{l}\text { - Subsecretaría de Recursos Hídricos } \\
\text { - Autoridad del Agua (ADA) } \\
\text { - Dirección Provincial de Saneamiento y Obras Hidráulicas } \\
\text { (2003-2015) / Dirección Provincial de Hidráulica (2015-2019) } \\
\text { - Dirección Provincial de Agua y Cloaca (DIPAC) } \\
\text { - Organismo de Control de Agua (OCABA 2003-2018) } \\
\text { - Servicio Provincial de Agua Potable y Saneamiento Rural } \\
\text { (SPAR 2003-2018) } \\
\text { - Autoridad de Cuenca Matanza Riachuelo (ACUMAR) }\end{array}$ \\
\hline
\end{tabular}

Fuente: Elaboración propia. 


\section{Reflexiones finales}

En Argentina, así como en América Latina en general, son aún insuficientes los desarrollos teóricos que relacionen el OT y la gestión del agua (y en particular con la GIRH), y los estudios que analicen comparativamente estas políticas. En ese sentido, el presente trabajo constituye un avance en el análisis de la vinculación de los aspectos normativos e institucionales del OT y la gestión del agua, en particular, del proceso de GIRH en Argentina y la PBA. Asimismo, aporta un marco de referencia útil para avanzar en el estudio de esta vinculación.

No obstante, en estudios futuros será fundamental profundizar en las perspectivas y prácticas no sólo de los actores sociales públicos, sino también de los agentes privados y de la sociedad civil que intervienen a lo largo del ciclo de las políticas públicas.

Los antecedentes normativos e institucionales analizados en este trabajo invitan a profundizar en los desafíos que supone la implementación de las políticas públicas, tales como: una concepción histórica desde perspectivas sectoriales; una discontinuidad en las políticas asociada a los cambios de gobierno; un elevado grado de fragmentación territorial e institucional; débiles marcos legislativos y regulatorios; y objetivos, estrategias y mecanismos de seguimiento de políticas poco claros (Organización para la Cooperación y el Desarrollo Económicos [OCDE], 2012).

Para concluir, la indagación realizada permite destacar la importancia de articular el OT y la gestión hídrica desde una perspectiva integral que trascienda los límites jurisdiccionales a fin de contribuir a la sostenibilidad de los territorios abordados en el marco de las metas previstas en los ODS.

\section{Agradecimientos}

Los/as autores/as agradecen a la Universidad Nacional de Mar del Plata (UNMdP) por el financiamiento recibido a través de su programa de subsidios a proyectos de investigación, al igual que a la Agencia Nacional de Promoción Científica y Tecnológica (ANPCyT) a través del Proyecto de Investigación Científica y Tecnológica PICT 2018-1682.

\section{Referencias bibliográficas}

ANDRADE PÉREZ, A. \& NAVARRETE LE BLAS, F. Serie Manuales de Educación y Capacitación Ambiental 8. Lineamientos para la aplicación del enfoque ecosistémico a la gestión integral del recurso hídrico. Ciudad de México: PNUMA, 2004.

BISWAS, A.K. Integrated Water Resources Management: Is It Working? International Journal of Water Resources Development, 2008, Vol. 24, Nº1, p. 5-22.

CARMAN, M. La fabricación de una comunidad moral. El caso de los afectados de la causa Matanza-Riachuelo. EURE, 2019, Vol. 45, №135, p. 111-130. 
CARTER, J.G. Spatial planning, water and the Water Framework Directive: insights from theory and practice. The Geographical Journal, 2007, Vol. 173, N4, p. 330-342.

CLICHEVSKY, N. Serie Medio Ambiente y Desarrollo No 75: Pobreza y acceso al suelo urbano. Algunos interrogantes sobre las políticas de regularización en América Latina. Santiago de Chile: CEPAL, 2003.

CORTI, M. El Decreto-Ley 8.912/77 de Ordenamiento Territorial y Uso del Suelo en la Provincia de Buenos Aires. Un análisis crítico de la legislación argentina. El Café de las Ciudades, 2009, Vol. 8, No85.

DELGADILLO, O. \& DURÁN, A. La cuenca hidrosocial: Una aproximación conceptual y metodológica para la gestión del agua en cuencas. En: F. QUIROZ; O. DELGADILLO \& A. DURÁN (Eds.). Aguas arriba, aguas abajo. Luces y sombras de la gestión integral de los recursos hídricos: Reflexiones desde la investigación aplicada. La Paz, Bolivia: Plural editores, 2012, p. 81-134.

DEL MORAL ITUARTE, L. Nuevas tendencias en gestión del agua, ordenación del territorio e integración de políticas sectoriales. Scripta Nova: Revista Electrónica de Geografía y Ciencias Sociales, 2009, Vol. 13, N²85.

DIRECCIÓN PROVINCIAL DE SANEAMIENTO Y OBRAS HIDRÁULICAS. La planificación y la gestión de los recursos hídricos debe ser una política de estado. La Plata: Ministerio de Infraestructura de la Provincia de Buenos Aires, 2009.

DOUROJEANNI, A. \& JOURAVLEV, A., Serie Recursos Naturales e Infraestructura, No 35: Crisis de gobernabilidad en la gestión del agua. (Desafíos que enfrenta la implementación de las recomendaciones contenidas en el Capítulo 18 del Programa 21). Santiago de Chile: División de Recursos Naturales e Infraestructura/CEPAL, 2001.

DOUROJEANNI, A.; JOURAVLEV, A. \& CHÁVEZ, G. Serie Recursos Naturales e Infraestructura, No 47: Gestión del agua a nivel de cuencas: teoría y práctica. Santiago de Chile: División de Recursos Naturales e Infraestructura/CEPAL, 2002.

DUEK, A.E. \& COMELLAS, E. Ordenamiento territorial y gestión integrada de los recursos hídricos: dos políticas implementadas en Argentina. Revista Tiempo y Espacio, 2011, Vol. 26, p. 153-170.

GARCÍA P.; BADANO, N.; MENÉNDEZ, A.; BERT, F.; GARCÍA, G.; PODESTÁ, G.; ROVERE, S.; VERDIN, A., RAJAGOPALAN, B. \& ARORA, P. Influencia de los cambios en el uso del suelo y la precipitación sobre la dinámica hídrica de una cuenca de llanura extensa. Caso de estudio: Cuenca del Río Salado, Buenos Aires, Argentina. Ribagua, 2018, Vol. 5, №2, p. 92-106.

GUDIÑO, M.E. \& CUELLO RÜTTLER, L. Derecho al agua. Aportes del ordenamiento territorial a la gestión y planificación de los recursos hídricos. En: M. PINTO; J. ESTRELLA \& A. GENNARI. Agua y Sociedad. CABA: Lajouane, 2017. 
GUTIÉRREZ, R.A. Modelo para armar: gestión del agua en la provincia de Buenos Aires. En: Sexto Congreso Argentino de Administración Pública. Resistencia: Asociación Argentina de Estudios de Administración Pública, 2011.

GLOBAL WATER PARTNERSHIP (GWP). TAC Background Papers N4: Manejo integrado de recursos hídricos. Estocolmo: GWP, 2000.

GLOBAL WATER PARTNERSHIP (GWP). Manual para la Gestión Integrada de los Recursos Hídricos. Londres: GWP y International Network of Basin Organizations (INBO), 2009.

JOURAVLEV, A. Serie Recursos Naturales e Infraestructura, No 27: Administración del agua en América Latina y el Caribe en el umbral del siglo XXI. Santiago de Chile: División de Recursos Naturales e Infraestructura/CEPAL, 2001.

KIDD, S. Towards a framework of integration in spatial planning: an exploration from a health perspective. Planning Theory \& Practice, 2007, Vol. 8, №2, p. 161-181.

MASSIRIS CABEZA, A. Gestión territorial y desarrollo. Hacia una política de desarrollo territorial sostenible en América Latina. Tunja: Universidad Pedagógica y Tecnológica de Colombia, 2012.

MOLLE, F. La gestion de l'eau et les apports d'une approche par la political ecology. En: D. GAUTIER \& A.T. BENJAMINSEN (Eds.), L'approche Political Ecology: Pouvoir, savoir, environnement, Paris: Quae, 2012, p. 219-238.

MOLLE, F. Planning and Managing Water Resources at the River-Basin Level: Emergence and Evolution of a Concept Colombo: International Water Management Institute, 2006.

MOLLE, F. \& WESTER, P. River Basin Trajectories: an Inquiry into Changing Waterscapes. En F. MOLLE \& P. WESTER (Eds.). River Basin Trajectories: Societies, Environments and Development. Londres: CABI/International Water Management Institute, 2009, p. 1-19.

ORGANIZACIÓN PARA LA COOPERACIÓN Y EL DESARROLLO ECONÓMICOS (OCDE). Gobernabilidad del Agua en América Latina y el Caribe: Un enfoque multinivel. París: Éditions OCDE, 2012.

OSZLAK, O. \& O’DONNELL, G. Estado y políticas estatales en América Latina: hacia una estrategia de investigación. CABA: Centro de Estudios de Estado y Sociedad (CEDES), CLACSO, 1981.

OVALLES, Y.; MÉNDEZ VERGARA, E. \& RAMÍREZ, G. Ordenación de cuencas hidrográficas. Un reto al conocimiento, la acción y la gestión. Revista Forestal Venezolana, 2008, Vol. 52, N², p. 241-252.

POCHAT, V. Principios de GIRH. Bases para el desarrollo de planes nacionales. Estocolmo: GWP-Central America y GWP-South America, 2008.

POTOCKO, A. La cuenca del río Reconquista en la planificación metropolitana de Buenos Aires (Argentina). Ámbito, problemas y propuestas. Urbe, Revista Brasileira de Gestão Urbana, 2017, Vol. 9, No3, p. 443-455. 
RíOS, L.; ROCCA, M.J.; \& BONO, N.O. Desarrollo local y ordenamiento territorial en la Provincia de Buenos Aires. Revista Geografar, 2008, Vol. 3, N², p. 59-85.

RUIZ, P.; PONCE, N. \& ARAOZ, L. Habitar entornos con riesgo hídrico. Vivienda colectiva y espacios productivos en el Arroyo Maldonado. Estudios del Hábitat, 2019, Vol. 17, N².

THWAITES REY, M. Capítulo 1: El Estado: Notas sobre su(s) significado(s). En: M. THWAITES REY \& A. LÓPEZ (Eds.). Entre tecnócratas globalizados y políticos clientelistas. Derrotero del ajuste neoliberal en el Estado argentino. CABA: Prometeo, 2005.

VICH, A.I.J., 2010. El sistema hídrico como determinante del ordenamiento territorial. Curso Gestión Integrada de los Recursos Hídricos en Acción, Maestría en Gestión Integrada de los Recursos Hídricos. Mendoza: UNCuyo y CELA-INA, 2010.

WIERING, M. \& IMMINK, I. When water management meets spatial planning: a policy-arrangements perspective. Environment and Planning C: Government and Policy, 2006, Vol. 24, p. 423-438.

WOLTJER, J. \& AL, N. Integrating Water Management and Spatial Planning. Journal of the American Planning Association, 2007, Vol. 73, N², p. 211-222.

WWAP (Programa Mundial de Evaluación de los Recursos Hídricos de la UNESCO). Informe Mundial de las Naciones Unidas sobre el Desarrollo de los Recursos Hídricos 2019: No dejar a nadie atrás. París: UNESCO, 2019. 
